\title{
Observations of Non-radial Pulsations in Radio Pulsars
}

\author{
J. Christopher Clemens ${ }^{1} \&$ R. Rosen \\ Department of Physics and Astronomy, University of North Carolina, Chapel Hill, NC \\ 27599-3255 \\ clemens@physics.unc.edu; rrosen@physics.unc.edu
}

\begin{abstract}
We introduce a model for pulsars in which non-radial oscillations of high spherical degree $(\ell)$ aligned to the magnetic axis of a spinning neutron star reproduce the morphological features of pulsar beams. In our model, rotation of the pulsar carries a pattern of pulsation nodes underneath our sightline, reproducing the longitude stationary structure seen in average pulse profiles, while the associated time-like oscillations reproduce "drifting subpulses" - features that change their longitude between successive pulsar spins. We will show that the presence of nodal lines can account for observed $180^{\circ}$ phase jumps in drifting subpulses and their otherwise poor phase stability, even if the time-like oscillations are strictly periodic. Our model can also account for the "mode changes" and "nulls" observed in some pulsars as quasiperiodic changes between pulsation modes of different $(\ell)$ or radial overtone $(n)$, analogous to pulsation mode changes observed in oscillating white dwarf stars. We will discuss other definitive and testable requirements of our model and show that they are qualitatively supported by existing data. While reserving judgment until the completion of quantitative tests, we are inspired enough by the existing observational support for our model to speculate about the excitation mechanism of the non-radial pulsations, the physics we can learn from them, and their relationship to the period evolution of pulsars.
\end{abstract}

Subject headings: pulsars:individual:PSR1237+25 — pulsars:individual:PSR1919+21— pulsars:general — stars:neutron — stars:oscillations

\footnotetext{
${ }^{1}$ Alfred P. Sloan Research Fellow
} 


\section{INTRODUCTION}

Upon the discovery of radio pulsations from pulsars by Hewish et al. (1968), Ruderman (1968) proposed that the pulses arose from non-radial oscillations of a neutron star. This idea was quickly displaced by a rotational model (Gold 1969), but Drake \& Craft (1968) again raised the possibility of pulsations when they measured individual pulse sequences for two pulsars and found within them narrow subpulses that moved to successively earlier times within the main pulse. Because this drift represented the presence of a "second periodicity" incommensurate with the spin period, it was natural to propose a time-like oscillation of the star. Subsequent measurements, however, revealed complex subpulse patterns that did not conform to a pulsation model in any obvious way. Moreover, the persistence of unique subpulse shapes from pulse to pulse, along with problems of phase stability we will address in later sections, led Drake to conclude that the drifting subpulses were incompatible with the pulsation hypothesis (see Staelin et al. 1970; Hewish 1970). Ultimately, pulsations were abandoned in favor of purely geometric models, although they reappeared from time-to-time in the theoretical literature (notably Hansen \& Cioffi 1980; van Horn 1980; McDermott, van Horn, \& Hansen 1988; Carroll et al. 1986; Finn 1990; Reisenegger \& Goldreich 1992; Strohmayer 1993). Most recently Duncan (1998) invoked toroidal modes to account for oscillations of soft gamma repeaters, but other than the work of Strohmayer (1992) and Strohmayer, Cordes, \& van Horn (1992), there has been no determined attempt to account for the properties of classical pulsars with models involving non-radial pulsations.

Instead, most current models, though not all (cf. Lyne \& Manchester 1988; Han \& Manchester 2001), incorporate a circulating pattern of sub-beams, whose motion about the magnetic pole produces the drifting subpulses. In these models, pulsar emission comes from accelerated particles that originate near the pulsar magnetic pole and travel along curved paths in the star's magnetic field (see Radhakrishnan \& Cooke 1969; Komesaroff 1970). The radiation is confined to a narrow beam by the dipole magnetic field geometry (Goldreich \& Julian 1969) and relativistic beaming along the direction of particle motion, which is roughly parallel to the magnetic axis, not perpendicular as in the models of Gold (1969), Smith (1970) and Zheleznyakov (1971). The observed brightness of pulsar beams effectively demands that the radiation is coherent, but the question of how it is produced is not settled (Jessner, Lesch, \& Kunzl 2001; Lesch et al. 1998; Melrose 1995).

Early studies of pulsar single pulses and average pulse shapes (Taylor, Manchester, \& Huguenin 1975; Lyne, Smith, \& Graham 1971, and others) led to the addition of more elaborate emission structures within the model pulsar beam. These features sweep past our

sightline and recreate the variety of pulse shapes we observe. Backer (1976) described a target-shaped emission pattern (a central core surrounded by an annulus) that can reproduce 
a wide variety of pulse morphologies depending on whether our sightline crosses the center of the pattern, yielding a three component pulse, or crosses only the annulus, resulting in a one or two component pulse. Oster \& Sieber (1977) added a second annulus and rotating features to reproduce pulses with more than three components and drifting subpulses. In 1975, Ruderman \& Sutherland (1975) supplied a physical basis for the model by suggesting that the emission arises from localized discharges or sparks near the polar cap. These are arranged in annular patterns, and rotate naturally due to the crossed components of the magnetic and electric fields.

In addition to the fixed and drifting substructure, models must account for observations of two kinds of discrete events observed in some pulsars; "mode changes", which abruptly alter the character of the substructure, and pulse "nulling", during which the pulse emission drops below detectable levels for one or more spin periods of the pulsar (Backer 1970a,b; Bartel et al. 1982). In the Ruderman \& Sutherland (1975) model, mode changes and nulling result from a collapse or reorganization of the fixed and moving spark structures, after which they must reappear with the same features they had previously.

Several reviewers have summarized observational and theoretical progress in the study of pulsar beams. The most ambitious is Rankin (Rankin 1983a,b, 1986, 1990; Radhakrishnan \& Rankin 1990; Rankin 1993a; Mitra \& Rankin 2002), who has both reviewed and synthesized the observations into an empirical model incorporating polarization and spectral behavior. Manchester (1995) gives a somewhat different view of the beam geometry. Most recently, Graham-Smith (2003) has published a succinct review that includes both "normal" and millisecond pulsars.

Against this backdrop, as a student project, we conducted a re-analysis of archival data on PSR0943+10 to look for evidence of non-radial pulsations, which, according to theory, might have periods ranging from milliseconds to seconds (McDermott, et. al 1988). Our analysis, which will appear in a subsequent paper, convinced us that time-like oscillations with a period of $31.8 \mathrm{msec}$ are a viable alternative to the rotating carousel of emission beams proposed by Deshpande \& Rankin (2001), but we could find no compelling reason other than aesthetics to prefer a pulsational model. In search of a definitive test, we reviewed the extensive observational literature on pulsars, and found intriguing evidence for non-radial pulsations as a universal mechanism for drifting and stationary subpulses. Moreover, we found that the original reason for abandoning pulsational models does not apply to nonradial pulsations of high azimuthal degree $(\ell)$ in which our sightline crosses pulsation nodal lines. The presence of nodal lines increases the variety and subtlety of expected subpulse behavior.

The purpose of this paper is to introduce a model in which high $\ell$ pulsations aligned to 
the pulsar magnetic pole take the place of the fixed and moving structures of the circulating spark model, but other details of the geometry remain unchanged. In this paper we will explore only the phenomenological consequences of this substitution, and compare them qualitatively to published observations. We will not discuss in any detail problems in the physics of pulsed radio emission or polarization mechanisms. In $\S 2$, we will present the basic features of our model, and explore its observational properties, some of which are not immediately obvious. Our main purpose is to lay the groundwork for future application of the model to radio measurements of individual and average pulse profiles. In $\S 3$ we will examine qualitative evidence in favor of our model, reserving quantitative comparisons for subsequent papers. The strongest evidence we will present comes from published measurements that show subpulse phase behavior difficult or impossible to explain using the circulating spark model, but demanded by high $\ell$ pulsations. We will also discuss analogies between pulsar behavior and that of known pulsating stars, specifically the rapidly oscillating peculiar A stars (roAp) and the pulsating white dwarf stars. This will demonstrate that there are precedents for the model behavior we propose. In $\S 4$, we will speculate about theoretical aspects of our model, such as the pulsation driving mechanism, and we will introduce the notion of "horizontal mode trapping", which can account for the high $\ell$ character of the proposed modes and relate them to the observed period evolution of pulsar beam widths. We will end by highlighting the potential for neutron star seismology, which can yield direct measurements of interesting physical quantities like the buoyancy of neutron star surface oceans.

\section{MORPHOLOGY OF NON-RADIAL PULSATIONS}

In this section we will describe the basic emission patterns that we expect non-radial pulsations to produce. It is simplest, though not strictly necessary, to confine ourselves to pulsations where the material displacements follow spherical harmonics with azimuthal order $m=0$. Using the notation Robinson, Kepler, \& Nather (1982) applied to white dwarfs, we can express displacements as follows:

$$
\xi=Y_{\ell, 0}(\Theta, \Phi) \cos \left(w_{t} t+\phi\right)
$$

Where the $Y$ is a spatial distribution of pulsation amplitudes, $\Theta$ and $\Phi$ are spherical coordinates aligned to the magnetic axis of the star, and $\cos \left(\omega_{t} t+\phi\right)$ is a time-like variation. 


\subsection{Fixed and Variable Pulse Structure}

Already we see in equation 1 the expression of an important feature of pulsar emission. In a series of individual pulses from a pulsar there may be no two alike, yet the average of a sufficient number of pulses builds up a profile that is stable in longitude and repeatable. Figure 1 illustrates this behavior with two sequences of pulses from pulsar PSR0943+10 and their respective averages. A series of "driftbands" that represent the positions of subpulses in successive spins of the pulsar, can be seen going from right to left. Note that within each individual pulse there are two, sometimes three, subpulses, but the average pulse shape is single-peaked. The abscissa is actually time within the pulse, but as conventional we plot longitude calculated according to the formula $\Phi=360^{\circ} / P_{1}$, where $P_{1}$ is the pulsar spin period. Following standard convention, we will use $P_{2}$ to represent the time interval between consecutive subpulses (the horizontal spacing between subpulses in figure 1) and $P_{3}$ to represent the time required for subpulses to return to a fiducial longitude (the vertical spacing between subpulses in figure 1).

Though they exhibit large pulse-to-pulse amplitude variations, the drifting pulses in figure 1 are modulated, on average, by a longitude stationary envelope. In some pulsars this has more complex structure than in PSR0943+10, as we shall see in a moment. In her review and synthesis of pulsar data, Rankin (1983a) expressed the difference between the information carried by average pulsar profiles and sequences of individual pulses thus: "it seems that profiles and pulse sequences must then each manifest some largely independent physical basis in the emission region." As seen in equation 1, non-radial oscillations offer a natural separation between fixed and variable structure in the form of spatial and time-like portions of a normal oscillation mode.

In addition to oscillations obeying equation 1, we must also propose that these oscillations are coupled to the radio emission mechanism, and that they can generate timemodulated emission according to $\xi$ of equation 1. For consistency with the observations, we do not want the pulsations to subtract emission in the negative part of their cycle, so in our simulations we have kept only positive values of $\xi$. An alternative approach, analogous to the method of Edwards \& Stappers (2002), would be to add a longitude-dependent bias to

Fig. 1.- Two sequences of 100 pulses each from $430 \mathrm{Mhz}$ observations of PSR0943+10 (lower panels), and their averages (upper panels). The subpulses show organized drift from right to left, along with disorganized amplitude behavior, but their averages converge to similar envelopes. The data are from Suleymanova et al. (1998) and are also published in Deshpande \& Rankin (2001). 
$\xi$. This would change the appearance of the simulated individual and average pulse shapes, and if large enough, would mute the nodal structure in the average pulse shapes relative to those shown in this paper.

We also assume that for a fixed radio frequency band, the emission originates at about the same altitude above the magnetic pole. This means that longitudinally distinct regions on the stellar surface will correspond to longitudinally separated pulse components, though the separation grows larger with increasing emission altitude due to the dipole field geometry (Komesaroff, Morris, \& Cooke 1970; Thorsett 1991). This assumption is consistent with the measurements of Gil (1991) and Gil \& Kijak (1992), who found similar emission heights for the various pulse components, but in conflict with the picture described by Rankin (1993a) or even Gangadhara \& Gupta (2001) (see also Gupta \& Gangadhara 2003). Finally, we note the sinusoidal pulses that result from equation 1 will not be sufficient to reproduce the nonsinusoidal profiles seen in figure 1, or the large variations in pulse size, but they will illustrate the essential morphological features pulsations can produce. This level of abstraction will allow later incorporation of simulated emission mechanisms (e.g. shot-noise models, Rickett 1975; Strohmayer et al. 1992) without affecting the tests of our model presented in this paper.

Figure 2 shows oscillations with $\ell=70$ and $m=0$ mapped onto the surface of a neutron star aligned with the magnetic axis. Dark regions indicate negative displacements and light regions positive ones. After a half cycle of the pulsations, the dark regions would be light and vice versa, but the nodal lines separating them would remain unchanged, except for rotation of the whole pattern about the rotation axis of the star (shown as a line extruding from the top of the sphere in figure 2). This model is similar to the "oblique pulsator" model developed for roAp stars by Kurtz (1982), except that $\ell$ is much higher here, and the pulsar only emits from a small region near the magnetic pole. We have indicated the boundary of the emission region in figure 2 with a circle around the magnetic pole. In $\S 4$ we will present a justification for why this boundary should coincide with a nodal line, and we will propose that the oscillations have different amplitude, perhaps even zero amplitude, outside of this boundary, a property we have not tried to reproduce in figure 2. Following convention, we will use $\alpha$ to denote the angle between the pulsar's spin and magnetic axes, and $\beta$ for the minimum angular separation between the magnetic pole and our line of sight, which is sometimes also called the "impact parameter".

If the pulsar in figure 2 rotates such that the emission region passes under our line of sight, we can observe two different kinds of variations. Because the oscillation amplitude is always zero at nodal lines, but can be non-zero elsewhere, the nodal lines sweeping past our line of sight can create pulses with a repetition rate related to the rate of nodal line passage. 
To estimate this rate, consider the case $\alpha=90, \beta=0$. In one full spin of the star our sightline crosses each nodal line twice, so the crossing rate is $P_{1} / 2 \ell$, but the period of a full cycle of the variations is twice this amount, or $P_{1} / \ell$, because $\xi$ changes sign at each nodal line. For arbitrary $\alpha$, the number of crossings is reduced by $\sin (\alpha)$, so the apparent average period of the spatial variations is:

$$
P_{\text {node }}=\frac{P_{1}}{\ell \sin (\alpha)}
$$

For simplicity, we have suppressed the more complicated dependence on $\beta$, which can be seen in the inset of figure 2 . The important feature to recognize is that the zeroes caused by the spatial node pattern remain at fixed longitude in subsequent spins of the pulsar unless either $\ell$ or the emission geometry changes.

At the same time as these nodal lines sweep past, the time-like oscillations generate pulses with a repetition rate related to the oscillation frequency as follows:

$$
P_{\text {time }}=\frac{2 \pi}{\omega_{t}}
$$

The behavior we observe in a pulsar beam depends upon the relationship between these two periods. If $P_{\text {node }}>P_{\text {time }}$, then we will see subpulses narrower than the nodal line structure, and, as long as $P_{\text {time }}$ is incommensurate with $P_{1}$, these subpulses will drift in longitude. Furthermore, as long as the measurement does not span a nodal line, the separation between subpulses $P_{2}$ will be approximately equal to $P_{\text {time }} . P_{2}$ is not exactly $P_{\text {time }}$ because the nodal structure that modulates the amplitudes of the subpulses also affects their times-of-maxima. For $P_{\text {node }}>P_{\text {time }}$, this causes longitude dependent subpulse drift such that $P_{2}$ is less than $P_{\text {time }}$ near nodal lines. In Appendix A we quantify this behavior and show examples of the driftband curvature it generates.

Fig. 2.- An oblique pulsator model for pulsar beams, showing an $\ell=70, m=0$ spherical harmonic aligned to the magnetic axis of a neutron star. The angle between the rotation axis and magnetic pole is $\alpha=50^{\circ}$ in this illustration. The circle around the magnetic pole in the enlarged view denotes the boundary of the emitting region. This region is crossed by four sightlines with different impact parameters ( $\beta$, see text). For each sightline, the inset shows the corresponding rectified slice of the spherical harmonic, representing the average beam profile. At the boundaries corresponding to nodal lines, subpulse phase changes by $180^{\circ}$, denoted by alternating + and - signs in the figure. 
In contrast to the appearance of individual pulses, the average of a sufficiently large number of pulses will reveal the fixed nodal line structure. In the inset of figure 2, we have shown what this nodal line structure would look like by plotting various traverses our sightline might make across the magnetic pole. For each traverse, we have plotted a rectified spherical harmonic to simulate the average of many spin periods where emission occurs only when $\xi$ is positive in equation 1. Our figure is intentionally similar to that of Backer (1976), but whereas the spacing and width of his annular features was arbitrary, ours follows the spacing and shape of spherical harmonics. We will return to this and other features after considering the case where $P_{\text {node }}<P_{\text {time }}$.

For $P_{\text {node }}<P_{\text {time }}$, individual pulses show no structure significantly narrower than the nodal line spacing, but the modulation of fixed pulse components at $P_{\text {time }}$ can still generate quasi-stationary driftlike variations. We have described the approximate behavior of pulse maxima for $P_{\text {node }}<P_{\text {time }}$, in Appendix A, and have shown an example of synthetic data for this case in the right hand panel of figure 3. Measurements of $P_{2}$ from a single pulse in this case will be strongly affected by $P_{\text {node }}$, making it difficult to estimate $P_{\text {time }}$ without modeling. In spite of these differences, the average of a large number of pulses will look the same as in the case previously discussed, and as simulated in the inset of figure 2.

For both cases, there is a $180^{\circ}$ shift in subpulse phase between pulse components separated by a nodal line. This has been indicated by alternating + and - signs in the inset of figure 2. This means that the driftbands caused by drifting subpulses, like those in figure 1, will not be continuous across nodal lines. We have simulated this behavior in figure 3 . The left hand panel shows a model representing PSR0943+10, where the sightline traverse resembles the $\beta=3.4^{\circ}$ case (with outer components missing), or the $\beta=5.7^{\circ}$ case shown in figure 2. No nodal line is crossed, and the drift is continuous across the whole profile. On the right is a model representing the 5-component profile of PSR1237+25, whose impact parameter is smaller. For this model, adjacent pulse components have different driftband phase, so there is no continuous pattern extending across the profile. The model we have used to represent PSR1237 +25 also has $P_{\text {node }}<P_{\text {time }}$, and illustrates the nature of the drifting in

Fig. 3.- Oblique pulsator simulations representing individual and average pulse profiles of PSR0943+10 (left) and PSR1237+25 (right). The PSR0943+10 simulation uses $\ell=83$, $\alpha=11.5^{\circ}, \beta=5.4^{\circ}, P_{1}=1.098 \mathrm{~s}$, and $P_{\text {time }}=31.78 \mathrm{msec}$. There are no nodal lines in the pulse window, and $P_{\text {time }}<P_{\text {node }}$, so subpulses appear to drift continuously across the profile. The PSR1237 +25 simulation uses $\ell=85, \alpha=53^{\circ}, \beta=0^{\circ}, P_{1}=1.382 \mathrm{~s}$, and $P_{\text {time }}=89.90$ msec. There are four nodal lines in the pulse window, and $P_{\text {time }}>P_{\text {node }}$ so subpulses appear as quasi-stationary variations with phase reversals at the nodal lines. 
that case.

\subsection{Requirements of the Model}

The model properties described so far are broadly consistent with the observed behavior of pulsar radio emission, but to focus the discussion onto specific tests, we will state as succinctly as possible three definitive requirements of the pulsation model for comparison with observations.

1. At the nodal line separating adjacent pulse components, subpulse amplitudes should be zero, and their phase should jump by $180^{\circ}$.

This assumes that only one pattern of nodal lines is present at a time, an assumption that could be violated if several pulsation eigenmodes are excited simultaneously, as occurs in the white dwarf stars. Note that 1 does not require the radio emission be zero at nodal lines, but rather that the modulated component of the emission be zero; we have not explicitly required that all of the emission come from the pulsations. As a corollary to requirement 1 , subpulse phase should drift almost linearly between the $180^{\circ}$ jumps, to within the effects of relativistic aberration and delay (see Gil 1991). If, however, the subpulse phase is inferred from the times-of-maxima of individual subpulses, these will follow the curvature calculated in Appendix A. The literature on driftband curvature (e.g. Wright 1981; Krishnamohan 1980) does not account for the possibility of $180^{\circ}$ phase jumps, but we will show in $\S 3$ that they have been observed in a number of stars, most recently and dramatically by Edwards, Stappers, \& van Leeuwen (2003).

2. The spacing between fixed pulse components should follow the same distribution as a spherical harmonic sampled along a single sightline.

This requirement has to incorporate the effects of $\beta$, which is the first of several complications. The second complication lies in the radio frequency dependence of average profiles, which is far from simple (Mitra \& Rankin 2002). It is possible to understand these profile dependencies in the context of a radius to frequency mapping model, as first proposed by Komesaroff, Morris, \& Cooke (1970) and explored by Thorsett (1991). In this model, lower frequency observations measure emission from a higher altitude, where the dipole field has diverged more. Since the emission is apparently tangent to the magnetic field, this divergence introduces a frequency dependent "magnification". This magnification broadens pulse 
components at low frequencies compared to their higher frequency counterparts, and changes the $\beta$ of effective sightline, since the particles emitting at higher altitude originated closer to the magnetic pole. However, if the magnification follows a dipole scaling, the ratios between component spacings will be preserved. Another difficulty arises from the Gaussian shape (Kramer et al. 1994) of measured pulse components, whose half-widths will differ from the cosine-like nodal regions of our model. We can mitigate this by comparing our model to measurements of pulse component maxima instead of widths, when possible. Finally, the emission we see probably represents an integral over some area on the star, due to the finite radio bandwidth and perhaps divergence of the emission itself. Together these problems make definitive tests problematic, but we will show that the average beam geometries explored by Rankin (1990, 1993a), Gould (1994), and Gil, Kijak, \& Seiradakis (1993), are crudely compatible with the requirements of our model.

3. Within the same pulse component, subpulses follow the relationship

$$
\frac{1}{P_{3}}=\frac{1}{P_{\text {time }}}-\frac{n}{P_{1}}
$$

where $n P_{\text {time }} \approx P_{1}$.

This arises because $P_{3}$ is simply a beat between $P_{1}$ and $P_{\text {time }}$ in our model. This relationship is the same as that given by Staelin et al. (1970) for pulsational models, except we have substituted $P_{\text {time }}$ for $P_{2}$. As we have discussed, when $\ell$ is sufficiently large that one or more nodal lines appear in the observed pulses, $P_{2}$ is not necessarily a good estimator of $P_{\text {time }}$, thus we cannot rule out the existence of stable clock based solely on the measured irregularity of $P_{2}$. According to Staelin et al. (1970), one of the primary reasons for rejecting pulsational models for drifting subpulses was the relative instability of $P_{2}$ compared to $P_{3}$. In $\S 3$, we will answer this objection to pulsation models, thirty-four years late, by reproducing observations of PSR1919+21, the first pulsar discovered. We will see that a model satisfying requirement 3 can simultaneously exhibit variations in $P_{2}$ like those measured by Drake \& Craft (1968) and Backer (1970c).

As a corollary to 3 , neither $P_{\text {time }}$ nor $P_{3}$ should be affected by the radius-to-frequency mapping that broadens $P_{\text {node }}$ at lower radio frequencies. So while the components of an average profile grow farther apart when observed at low frequency, the time-like pulses will not. Once again, it is crucial to recognize that $P_{2}$ may not be a good estimator of $P_{\text {time }}$, especially when $P_{\text {time }}$ exceeds $P_{\text {node }}$. In that case measurements of $P_{2}$ can be dominated by the nodal line structure instead of $P_{\text {time }}$. As we will discuss in $\S 3$, measurements of the frequency dependence of $P_{2}$ show negligible frequency dependence for those pulsars where $P_{\text {time }}<P_{\text {node }}$, and an increasing frequency dependence as $P_{\text {node }}$ approaches $P_{\text {time }}$ (Izvekova et al. 1993; Gil et al. 2002), consistent with the requirements of our model. 


\section{COMPARISON TO OBSERVATIONS}

\subsection{Subpulse Phase Jumps}

A phase jump of $180^{\circ}$ is not subtle behavior, so if our model is correct then this property of drifting subpulses should have been observed repeatedly. Interestingly, the first measurement of phase differences between adjacent pulse components came relatively early, in Taylor et al. (1975), but its significance for pulsation models was not recognized or pursued. Taylor et al. (1975) constructed individual time series for each of the five pulse components in PSR1237+25, and cross-correlated them. With the exception of the central component, their analysis showed that components adjacent to each other in longitude have opposite subpulse phase (see their figure 11). This behavior required Oster \& Sieber (1977) to place the emission regions on their inner circulating carousel out of phase with those on the outer (see figure 13 of Oster \& Sieber 1977), and led Hankins \& Wright (1980) to propose a spiral emission pattern. In addition to PSR1237+25, Taylor et al. (1975) found similar anticorrelations for the components of PSR0329+54. Eleven years later, Prószyński \& Wolszczan (1986) applied the same analysis with better resolution to PSR1919+21, PSR0809+74, and PSR1237+25 (again). All three of these objects show behavior consistent with $180^{\circ}$ jumps in their subpulse phases in at least one radio band.

In addition to these four objects, Edwards, Stappers, \& van Leeuwen (2003) recently applied their two-dimensional fluctuation spectrum technique to PSR0320+39, and found dramatic evidence for phase and amplitude modulation like that expected at a nodal line. In figure 4, we have reproduced figure 3 of their paper, which was largely responsible for guiding us to the model we are proposing. As Edwards \& Stappers (2002) point out, the two-dimensional Fourier transform as they apply it makes use of all the phase information in the data to produce phase and amplitude envelopes with high signal-to-noise ratio even for modest quality data. In figure 4 , the phase envelope shows the $180^{\circ}$ phase shift we expect at a nodal line, and almost linear behavior in between (a $60^{\circ}{ }^{\circ}$ slope has been removed from the data).

Fig. 4.- Subpulse amplitude (upper panel) and phase (lower panel) envelopes for PSR0320+39, reproduced with permission from Edwards, Stappers, \& van Leeuwen (2003). The upper panel also shows the average pulse shape (dotted line). The subpulse amplitude envelope shows a minimum near zero at the same longitude as a $180^{\circ}$ shift in the phase envelope, consistent with the requirements of the oblique pulsator model. The phase envelope is plotted three times representing analysis via three different techniques. A $60^{\circ}{ }^{\circ}$ slope has been removed from the phases. 
At the same longitude as the phase shift, the subpulse amplitude is near zero, as required at a nodal line. This figure evokes comparisons to figure 14 of Kurtz, Shibahashi, \& Goode (1990), which shows a similar phase shift in the rapidly oscillating Ap star HR3831 as rotation changes the viewing geometry of the pulsation nodal structure. We note that our model requires symmetry in the pulse components which means that a second phase jump should appear in the profile of figure 4. In their subsequent paper Edwards \& Stappers (2003) detected such a jump near the right hand edge of the profile.

Edwards \& Stappers (2003) applied a similar analysis to PSR0809+74 at two frequencies, with results that are more challenging for our model. The phase envelopes do not appear to be linear, and there are abrupt phase shifts not equal to $180^{\circ}$. Since the emission we observe is an integral over some frequency range and perhaps over some area on the star, we speculate that abrupt changes can be "washed out" by these inherent limits to the longitude resolution, especially at low frequency where the pulse components change their appearance most rapidly (Thorsett 1991). Whether PSR0320+39 is the lucky exception or the norm will require more data to tell. At any rate we do not think the problems with PSR0809+74 should overwhelm our model, especially when compared to the elaborations these phase changes require in the drifting spark model (Edwards \& Stappers 2003), but caution and careful modeling will be required.

For PSR1919+21, the prototype of pulsars and of drifting subpulses, we have reproduced a longitude resolved cross-correlation map from Prószyński \& Wolszczan (1986) in the left hand panel of figure 5. This is a contour plot of the cross-correlation of the time series at each longitude with that at a reference longitude. The maxima occur at lags where the subpulse peaks align and minima where peaks align with troughs. For subpulses that drift uniformly from one side of the profile to the other, these diagrams should be crossed by bands of continuous slope proportional to the drift rate. Instead, we see sloping bands punctuated by two phase inversions, indicating that at those longitudes the subpulses abruptly change their phase.

To illustrate that pulsations can reproduce this behavior, we have simulated PSR1919+21

Fig. 5. - A comparison of longitude-resolved cross-correlation maps for PSR1919+21. The left panel shows the cross-correlation of $1420 \mathrm{MHz}$ time series data from each longitude with that at a reference longitude, reproduced with permission from Prószyński \& Wolszczan (1986). The right panel shows the cross-correlation map of simulated data using an oblique pulsator model with $\ell=100, \alpha=45^{\circ}, \beta=-2.35^{\circ}, P_{1}=1.337 \mathrm{~s}$, and $P_{\text {time }}=32.01 \mathrm{msec}$. The phase reversals at $\sim-30$ and $\sim-8$ msec correspond to the locations of nodal lines in the model. Solid contours correspond to positive correlations. 
with a model like that shown in figure 2 . The model has only five parameters, $\alpha, \beta, P_{1}$, $\ell$, and $P_{\text {time }}$, the values of which are listed in the caption to figure 5 . First we generated a synthetic light curve using the positive values of $\xi$ in equation 1 sampled along a sightline defined by $\alpha$ and $\beta$. For each time sample in the light curve, we changed the longitude by $\Delta \Phi=360^{\circ} \Delta t / P_{1}$, and the pulsation phase by $\omega_{t} \Delta t$, where $\Delta t$ is the time resolution. We eliminated data outside the observed pulse window, and constructed individual time series at each longitude. Finally, we cross-correlated these time series using the formula provided in Prószyński \& Wolszczan (1986), and produced the contour map shown on the right hand side in figure 5. We chose model parameters based on published values (except for $\ell$ ), in some cases adjusting them slightly to improve the fit, which was done by eye. The model parameters should be regarded as illustrative only; no attempt was made to measure the quality or uniqueness of the fit.

In figure 6, we show individual pulse profiles for our model of PSR1919+21, to emphasize the variations that occur in $P_{2}$ even though the pulsation frequency is constant. We have indicated two different measured values of $P_{2}$ similar to those given by Backer (1970c), neither of which is close to the input oscillation period of $32.01 \mathrm{msec}$. We conclude that the published measurements of PSR1919+21 are qualitatively consistent with a pulsational model incorporating a stable pulsation period and high $\ell$.

We performed a second simulation for PSR1237+25, with the results pictured in figure 7 . Once again the qualitative similarity is encouraging. In this star, more of the profile seems to be "missing" than in the previous one. Our model offers no ready explanation for these zones where the pulsed emission disappears, but it seems that the mechanism for generating pulsed emission fails. This failure could also explain the asymmetry in the profile of PSR0320+39 that we discussed in conjunction with figure 4. To compound the problem, Radhakrishnan \& Rankin (1990) report that the pulsed emission seldom appears in the core component, and the polarization there is different, neither of which has any obvious basis in our model. On the contrary, the zone at the pole of a spherical harmonic has the smallest area and therefore the largest pulsational displacements (to make the surface integral equal to those of other zones), so unless some mechanism saturates we expect larger amplitudes from the core.

We can put these issues aside pending deeper investigation of the emission mechanism,

Fig. 6. - Synthetic individual pulse profiles for PSR1919+21, generated using the same model as in figure 5. The left-hand side shows subpulse separations similar to those measured by Backer (1970c). The right-hand side shows a larger number of pulses, making the amplitude modulation by nodal lines more apparent. 
but observations like those described in Hankins \& Wolszczan (1987) offer a more direct challenge to our model. They analyzed PSR1918+19 using the same cross correlation technique as shown in figures 5 and 7 , and found much different behavior. There is evidence in that pulsar for different drift rates in each of the 3 components of the average profile. We see no obvious way to reproduce the diagrams of PSR1918+19 with a single pulsation frequency, but we note that the time series were very short (just 27 pulses in one case) and the inclination $\alpha$ is unusually small. More measurements and detailed modeling may lead to a solution.

\subsection{Pulse Components and Their Separations}

Now we consider the spacing between pulse components in the average profiles of pulsars. If the components are related to the zones of a spherical harmonic, as we propose, then they cannot have arbitrary widths and separations. For example, in our pulsation model, the angular separation between the pulsation pole and the first nodal line is $\sim 0.44$ times the separation between the pole and second nodal line, independent of $\ell$ as long as $\ell$ is high. Similarly, first and second antinodes have angular separations from the pulsation pole in the ratio $\sim 0.55$. We expect the modulated pulse components in pulsars with $\beta=0$ to follow similar relationships. PSR1237+25 is an good example, since it has five components and Lyne \& Manchester (1988) and Rankin (1993b) give it $\beta \approx 0$ (as measured from rotation of the linear polarization angle). As an illustration, we have plotted in figure 8 the average profile of PSR1237 +25 along with a $\beta=0$ sightline through rectified spherical harmonic of $\ell=85$. Note that the actual $\ell$ at the surface of the pulsar will be higher, depending on how much magnification the dipole field geometry has imposed.

It would be much better, though quite difficult, to compare the spherical harmonics to a statistical sample of pulsars with known $\beta$. Fortunately, Rankin (1990, 1993a), Gould (1994), Gil, Kijak, \& Seiradakis (1993), Kramer et al. (1994), and Mitra \& Deshpande (1999) have studied the ensemble properties of pulse shapes and uncovered consistent ratios between the core and annular emission components after adjusting for or eliminating the effects of $\alpha$ and $\beta$. These ratios are statistical averages suitable for comparison to spherical harmonics, but first we will discuss their dependence on $P_{1}$.

Fig. 7.- A comparison of longitude-resolved cross-correlation maps for PSR1237+25. As in figure 5 the left panel shows a map reproduced from Prószyński \& Wolszczan (1986) for data at $408 \mathrm{MHz}$. The right panel shows the map for simulated data using an oblique pulsator model with $\ell=85, \alpha=53^{\circ}, \beta=0^{\circ}, P_{1}=1.382 \mathrm{~s}$, and $P_{\text {time }}=89.90 \mathrm{msec}$. 
Rankin (1990) found that the angular size of the central emission component or "core" follows a $P_{1}^{-1 / 2}$ dependence, when allowance is made for variations in $\alpha$. This dependence is the same as that Goldreich \& Julian (1969) calculated for the size of the polar cap delineated by open magnetic field lines, i.e. those lines that do not close within the velocity-of-light cylinder at $c P_{1} / 2 \pi$. In the Goldreich and Julian model, this cap is the region from which charged particles stream off the spinning pulsar, providing both a mechanism for shedding angular momentum, and the possibility for radio emission from above the magnetic polar cap. The $P_{1}^{-1 / 2}$ dependence of emission cores measured by Rankin suggests that they are related to this Goldreich-Julian polar cap, although we should note that Lyne \& Manchester (1988) measured a different $P_{1}$ dependence from Rankin.

In addition to the functional dependence, Rankin (1990) noticed that at any fixed $P_{1}$, the angular half width of pulsar emission cores was the same as the calculated apparent angular size of the Goldreich-Julian cap, e.g. about $2.5^{\circ}$ for $P_{1}=1 \mathrm{~s}$. This led her to suggest that the core emission fills the cap near the pulsar surface. While appealing in its simplicity, this explanation requires that the annular emission patterns originate at different heights along the last open field lines. Gil (1991) has criticized this suggestion on observational grounds, but provided no alternative physical reason for the emission core to follow the scaling of the magnetic cap. As we will discuss in $\S 4$, our model suggests an explanation if the boundary separating open and closed field lines can act as a "mode trapping" boundary, which is always coincident with a pulsation node. The size of the core region would then have quantized values that scale with $P_{1}$ according to the to size of the polar emission cap.

Based upon her conclusion that the emission core size depends only on $P_{1}$, and upon her measurements of the annular emission regions which show the same $P_{1}$ dependence, Rankin (1993a) was able to infer that the ratio of the angular sizes of the inner and outer emission cones is 1.32, as measured at the outer half-power points. In similar fashion, Gould (1994) measured half-widths for the core and annular zones and found that the core components are 1.4 times as wide.

If we compare these measured component ratios to analogous ratios for the components of a spherical harmonic observed along a $\beta=0$ sightline, we find that the ratio of the halfwidths of the core and annular components in the model is $\sim 1.49$ independent of $\ell$, or about $6 \%$ higher than the Gould measurement of 1.4. The ratio analogous to Rankin's inner and outer cone widths is not as good a match at $\sim 1.72,23 \%$ larger than Rankin's measurement. However, Gil, Kijak, \& Seiradakis (1993) have found evidence for a different angular size of

Fig. 8. - A comparison between a $\beta=0$ slice through a spherical harmonic of $\ell=85$ and the average pulse profile of $1237+25$ measured at $320 \mathrm{MHz}$ (see Rankin 1986). 
the inner components in five component pulsars, and the ratio of this innermost component with the outermost one is 1.62 , again about $6 \%$ below the 1.72 ratio measured in our model. In $\S 4$ we will discuss how our model might lead to more than one quantized value for the inner and outer annuli if different numbers of nodal lines are trapped within the polar cap. As we will discuss, there is evidence for this kind of quantization in the core component as well, but it has been interpreted as a preferred inclination angle $\alpha$ (Rankin 1990). For now, even if the results are somewhat ambiguous, it is gratifying to be able to make any testable predictions at all about the shape of average pulsar profiles.

\section{3. $\quad$ The Radio Frequency Dependence of Drifting Subpulses}

In the process of addressing model requirements 1 and 2 from $\S 2$, we have incidentally shown that the formula in requirement 3 is not necessarily contradicted by changes in $P_{2}$. Strict confirmation of requirement 3 will be difficult, because all attempts to measure $P_{\text {time }}$ are badly aliased by the narrow, periodic pulse window, although PSR0943+10 looks promising in the studies of Deshpande \& Rankin (2001). The best strategy may be to concentrate on the wider profiles measured when $\alpha$ is small, since the aliasing will not be as bad. Fourier methods, especially the two dimensional transform of Edwards \& Stappers (2002), will be indispensable to this effort, while direct measurements of $P_{2}$ in single or multiple pulses are misleading, as we have seen in our model of PSR1919+21.

The radio frequency dependence of $P_{2}$ is another area where the behavior of subpulse maxima can be misleading. Our model requires that $P_{\text {time }}$ and $P_{3}$ be invariant with radio frequency, but we have already seen that $P_{2}$ will vary near nodal lines, even when $P_{\text {time }}$ does not (see Appendix A). Consequently, since observing at a different frequency changes the apparent longitude of the nodal lines, $P_{2}$ can vary with radio frequency even though $P_{\text {time }}$ remains invariant. Qualitatively, we expect the radio frequency dependence of $P_{2}$ to approach zero when $P_{\text {time }}$ is much smaller than $P_{\text {node }}$ and no nodal lines are near. For $P_{\text {time }}$ approaching $P_{\text {node }}$, the change in $P_{\text {node }}$ with radio frequency will modulate $P_{\text {time }}$ as well and introduce a frequency dependence into $P_{2}$. In the limit of very large $P_{\text {time }}$, the radio frequency dependence approaches that of the average components.

Izvekova et al. (1993) have studied the frequency dependence of subpulses in four pulsars. They found that $P_{3}$ does not change with radio frequency, consistent with the requirement of our model. For $P_{2}$, the frequency dependence is in all cases less than that of the average profile, also consistent with our model. For PSR0031-07 and PSR1133+16, both of which have continuous driftbands across the profile, the frequency dependence of $P_{2}$ is very weak, $\sim \nu^{-0.05}$ and $\sim \nu^{-0.06}$ respectively, while the frequency dependence of the average 
profile is about $\sim \nu^{-0.3}$. The other two pulsars show larger frequency dependencies for $P_{2}$, but one of these is PSR0320+39, which we now know has multiple components separated by phase jumps, as shown in figure 4 . This means that the subpulse separation is similar to the separation between stationary profile components, and we expect a larger frequency dependence in this case. It will be interesting in future quantitative studies to attempt to reproduce the exact radio frequency dependence of the subpulses for individual pulsars by tuning $P_{\text {time }}$. In principle, each independent radio frequency measurement offers a separate constraint on $P_{\text {time }}$ that might be useful in verifying its stability with respect to $P_{3}$.

\section{4. $\quad$ Mode Changes and Nulls}

A number of pulsars exhibit abrupt changes in their drifting subpulse behavior (Rankin 1986) or their average pulse profiles (Bartel et al. 1982), or both. In some examples, these changes are cyclical or quasi-cyclical, such that the pulsar successively visits each of two or three modes (e.g. PSR0031-07). A much larger number of pulsars (Rankin 1986), including many of those with mode changes, undergo "nulls", in which the radio emission falls below detectable levels. The interesting property of these variations in the context of our pulsation model is the "memory" the pulsar must retain in order to return to the same states repeatedly. Inasmuch as pulsations represent eigenmodes of the neutron star, their eigenperiods reflect a durable physical structure that will vary only secularly as the star cools and slows down its spin. This means that a mode excited to observable amplitude and then damped can return again with essentially the same period.

Among the known pulsators, white dwarfs provide some exceptional examples of this behavior. The hydrogen atmosphere variable (DAV) white dwarf G29-38 sometimes oscillates with very large amplitudes in a dominant mode with $\sim 610$ s period, then changes abruptly to a large dominant mode at $\sim 809 \mathrm{~s}$, and then to very low amplitude pulsations with no dominant mode (Kleinman 1998). This mode changing behavior suggests the exchange of energy between eigenmodes with different amplitudes (for the same energy content), though this has not been established with certainty (Dziembowski 1982; Wu \& Goldreich 2001). We note the similarity of this behavior to pulsar mode changes and nulling. Pulsar mode changing involves changes in subpulse drift rates and in the mean profiles, both of which we might expect for changes between modes of different degree $\ell$. Likewise, changes in the subpulse drift rate only might correspond to changes in the radial eigennumber $n$. Furthermore, the exponential recovery of drift rates after nulls in PSR0809+74 (Lyne \& Ashworth 1983), suggests relaxation into a normal oscillation mode after a mode interaction. In the same star van Leeuwen et al. (2002) found that the pulsar is often, and maybe always, in 
a different drift mode immediately following a null, which shows that they two phenomena are physically related. While it is possible to interpret this in the drifting spark model (van Leeuwen et al. 2003), pulsation mode switching may offer a more natural explanation.

Finally, evidence that nulling and mode changing are global phenomena comes from the pulsars with interpulses PSR1822-09 and PSR1055-52. Fowler, Wright, \& Morris (1981) have observed that the interpulse emission in PSR1822-09 changes its intensity when the main pulse changes between burst and quiescent modes. Likewise, (Biggs 1990) found intensity correlations between the interpulse and main pulse in PSR1055-52, and suggested non-radial oscillation as a possible mechanism for communication between the poles.

\section{DISCUSSION}

Our main purpose in this paper has been to set forth the requirements of a model for pulsar beams in which non-radial oscillations of high $\ell$ replace the primum mobile of drifting sparks in the Ruderman \& Sutherland (1975) model. This groundwork will clarify future applications of our model to individual pulsars, which we will begin in a forthcoming study of PSR0943+10. Although quantitative investigations are required for definitive tests, we have presented observational evidence that our pulsation model should be an active contender for the attention of observers and theorists alike. We will continue with some theoretical speculation about the nature of the pulsations.

\subsection{The Nature of the Pulsations}

When we invoke non-radial oscillations in our model, we mean any oscillations in which time-like variations are accompanied by spatial nodal lines that rotation can carry past our sightline. Immediately, this suggests various deformations that might appear in the core, crust, or ocean of a neutron star (McDermott, et. al 1988), but we should not rule out other possibilities such as oscillations in the magnetosphere above the magnetic pole (e.g. Rylov 1978; Staelin et al. 1970; Schopper et al. 2002). The main problem we will encounter in identifying the pulsations is that most of the oscillations we can propose have frequencies too high to account for pulses with the repetition rate of $30 \mathrm{msec}$ typical for subpulse periods. To be fully general we also should not rule out high frequency oscillations that are "switched" at low frequency, but we will find little guidance in pulsar literature for models of this sort.

The two quantities that will assist us in identifying the kind of oscillations our model should include are $\ell$ and $P_{\text {time }}$. In our illustrations, we have used $\ell \approx 70-100$ to match the 
width of features in observed profiles, but these profiles apparently do not originate at the surface of the neutron star. Rather, they are magnified versions from radiation emitted at tangents to the diverging dipole field. We can estimate the magnification factor, which we will call $f_{\nu}$, by comparing the observed profile widths to the expected size of the GoldreichJulian emission cap at fixed period $P_{1}$. At 1 second, for a pulsar with radius $10 \mathrm{~km}$, the latter is $1.7^{\circ}$, while Rankin (1993a) measured $11.5^{\circ}$ for the width of the outer annular beam. Together, these yield a magnification of $f_{\nu} \sim 7$ at $1 \mathrm{GHz}$. Thus an apparent $\ell$ of 85 represents a true $\ell$ at the surface of $\sim 600$. The dispersion relationship for $g$-modes (and torsional modes) McDermott, et. al (1988) requires that $P_{\ell}$ scale as $1 / \ell$, so if $\ell=600$ has a period of $30 \mathrm{~ms}$, then we expect the $\ell=2$ mode with same radial overtone to have period $\sim 9 \mathrm{~s}$. This limits considerably the kinds of oscillations we might consider.

McDermott, et. al (1988) gives periods near 9 s for low $\ell$, low radial overtone $(n \approx 1)$, core $g$-modes. However, these modes require very large energies to excite, and are trapped in the core by the solid crust, yielding small amplitudes at the neutron star surface. An alternative from McDermott, et. al (1988) are the $g$-modes that propagate in the $\sim 1 \mathrm{~m}$ thick electron-degenerate Coulomb liquid ocean overlying the solid crust in equilibrium neutron star models (Richardson et al. 1982). The low $n$ ocean $g$-modes have periods near $0.3 \mathrm{~s}$, rather than the $9 \mathrm{~s}$ we require, but the dispersion relation for $g$-modes gives longer periods for higher overtone, so the eigenperiod should increase to $9 \mathrm{~s}$ for $n \approx 30$. Therefore, ocean $g$-modes of $\ell \approx 600$, and $n \approx 30$ match the $\ell$ and $P_{\text {time }}$ our model requires. According to McDermott, et. al (1988), these modes have lower energies than the core modes, and large surface amplitudes. As in white dwarf stars, the material displacements in these oscillations are primarily horizontal because of the high surface gravity.

The ocean $g$-modes also have an associated temperature variation that offers a way to modulate the flow of charged particles from the pulsar surface at the pulsation eigenperiod. If we accept the results of Jessner, Lesch, \& Kunzl (2001), the electrons accelerated along open field lines from a pulsar magnetic pole can be provided by thermal and field emission from the neutron star surface, without the formation of a vacuum gap where sparks originate in the Ruderman \& Sutherland (1975) model. This result practically requires that subpulses be related to a thermal variation at the neutron star surface, as $g$-modes provide. Thus non-radial oscillations appear to satisfy one of the basic requirements of a pulsar emission mechanism.

The other requirement for emission is that the liberated electrons be "bunched". Their acceleration away from the surface is naturally provided by the potential difference between the neutron star pole and the circum-pulsar medium (Goldreich \& Julian 1969), but conversion of the particle stream to coherent radiation requires bunching of charges, whether the 
conversion is via an antenna or a maser mechanism (Melrose 1995). The radial structure of $n=30$ ocean $g$-modes consists of $\sim 3 \mathrm{~cm}$ zones of alternating pulsation phase. At any instant, this will correspond to a periodic variation of the temperature and pressure with depth. In electron degenerate plasma, the temperature is a property of the ions while the pressure is a temperature insensitive property of the electrons. The displacements of an ion fluid element generate a buoyant restoring force only through their electrical coupling to the electrons, so the vertical pressure variation is associated with a vertically varying component of the electric field. If this can modulate the flow of electrons, as in a klystron, then the pulsations might also provide the mechanism for bunching the electrons emitted from the surface. The observational clues necessary to clarify this question may lie in subpulse polarization measurements, which are beyond the scope of this paper.

A problem with this picture comes from the work of Carroll et al. (1986), who added a strong magnetic field to the pulsation calculations. Because of the high electrical conductivity in the neutron star ocean, Carroll et al. (1986) treated the magnetic field as "frozen-in", and recalculated the pulsation frequencies for $B=10^{12} \mathrm{G}$ in the MHD limit. He found that the ocean $g$-modes become "magneto-gravity" modes with very short periods $(<1 \mathrm{~ms})$ and a different dispersion relation. The solution to this conundrum is provided by the conductivity calculations of Potekhin (1999). Potekhin finds electrical conductivities in the direction transverse to a $B=10^{12} \mathrm{G}$ field to be four to five orders of magnitude lower than the $10^{19} \mathrm{~s}^{-1}$ Carroll et al. (1986) assumed. Thus the ohmic diffusion timescale for

displacements of $10 \mathrm{~cm}$ or smaller is $\lesssim 1 \mathrm{msec}$, shorter than subpulse periods. Not only does this mean the calculations of Carroll et al. (1986) do not apply, it also means the magnetic field can simultaneously provide the driving mechanism, the amplitude limiting mechanism, and the mechanism for enforcing high radial overtones.

\section{2. $\quad$ Mode Driving and Trapping}

The high $\ell$ in our model suggests the concentration of pulsation driving energy into a small surface patch, otherwise it would average away in the sum over multiple surface zones with alternating phase. This concentration suggests consideration of the emission pole itself as the site of driving. The torque exerted by braking from particle emission will be concentrated at the open field lines, and communicated to the rest of the star by magnetic and mechanical dissipation. If the magnetic field is coupled (even weakly) to the surface and it displaces material laterally, the possibility for feedback and mode driving exists. For example, suppose that torque on the open field lines results in a displacement of material on the polar cap. The heating that results can increase particle emission, which increases the 
torque. Depending on the time delays, this feedback could drive oscillatory motion. Other possibilities involving direct shaking of field lines (Boriakoff 1976) by displaced material are also possible.

Whatever the driving mechanism, if there are $g$-mode pulsations in the neutron star ocean, it is reasonable to propose that their propagation behavior changes at the boundary between the emitting cap and the rest of the star, not only because the magnetic field changes from an open to closed configuration, but because the surface boundary condition changes. Thus the edge of the emitting cap constitutes a circular boundary that might "trap" pulsation modes in the horizontal direction, analogous to the trapping by composition transition zones in white dwarf stars (Winget, van Horn, \& Hansen 1981). This "horizontal mode trapping" could provide a connection between the size of the emitting cap and the size of pulsation nodes by forcing surface nodal lines to lie at the circular boundary. If this connection is maintained as $P_{1}$ increases, it can explain why the core emission zone, as measured by Rankin (1990) and others, follows a $P_{1}^{-1 / 2}$ relationship. In this section, we will explore pulsation period evolution in the context of a horizontal trapping model.

\subsection{The Period Evolution of the Pulsations}

The pulsars measured to date seldom, if ever, have more than five components in their average pulse (cf. Gangadhara \& Gupta 2001, although their methodology is compromised by pulsations) In our model, five components could result from horizontal mode trapping at the third nodal line from the pulsation pole, and this is the geometry we depicted in figure 2. We have already seen that parts of the profile can be "missing", so the presence of five components in the model does not necessarily mean that we see all five. If we now force $\ell$ to have a value that places the third node at the emission boundary, we can write a relationship for the period evolution of $\ell$. From Goldreich \& Julian (1969), the width of the emission cap for a $10 \mathrm{~km}$ star follows:

$$
W_{\text {cap }}=1.7^{\circ} P_{1}^{-1 / 2}
$$

and the width of third nodal line scales as $1 / \ell$, reaching $1.7^{\circ}$ for $\ell \approx 600$. So we may write:

$$
W_{3}=1.7^{\circ} \frac{600}{\ell}
$$

Enforcing horizontal mode trapping requires $W_{c a p}=W_{3}$ so, 


$$
\ell=600 P_{1}^{1 / 2}
$$

as long as the trapping stays at the third nodal line. This equation is a mathematical statement of the obvious requirement that as the emission cap shrinks during spin-down, the $\ell$ of trapped pulsations must go up. Some of the other consequences for the pulsations are

not as obvious. For instance, from the dispersion relation for $g$-modes,$P_{\text {time }}$ changes as $\sim 1 / \ell$ if the radial overtone doesn't change, so we expect $P_{\text {time }}$ to decrease as $P_{1}$ increases. However, the important ratio $P_{\text {time }} / P_{\text {node }}$ becomes:

$$
\frac{P_{\text {time }}}{P_{\text {node }}} \propto \frac{\sin (\alpha)}{P_{1}}
$$

So as $P_{1}$ increases (or the spin and magnetic axes align), $P_{\text {time }}$ shrinks with respect to $P_{\text {node }}$. This favors the appearance of narrow drifting subpulses in the long period pulsars, as observed. The impact parameter $\beta$, which we have ignored, may also play a role, since the decreasing emission cap size makes it less likely that our sightline intersects the central component (see figure 2), and $P_{\text {node }}$ increases for such sightlines.

Finally we consider the possibility that the horizontal trapping may apply to the second nodal line instead of the third, requiring a lower value of $\ell$ for the same $P_{1}$. In this case, the central component will have a larger angular size at the same $P_{1}$ than for third node trapping. This would be observed as a bimodal distribution of core components, and there is evidence in Rankin (1990) for just such an effect (see her figure 1). Because Rankin (1990) assumed that the distribution of core sizes is solely an effect of $\alpha$, the bimodality appears as an excess of pulsars at $\alpha=35^{\circ}$. While our explanation would eliminate this puzzling excess, it raises two problems of its own. First, we would expect some pulsars with interpulses in the distribution with larger core sizes, and Rankin (1990) finds none. Second, figure 1 of Rankin (1990) shows the beam sizes for pulsars with single components, not five, meaning all the pulsars in this sample have pulse components within their emission caps that do not appear in the mean profiles.

\section{CONCLUSIONS}

Whether or not the foregoing discussion has revealed anything about pulsars, it has certainly demonstrated Clemens (1883) maxim that we can get "wholesale returns of conjecture out of such a trifling investment of fact." Nevertheless, the fact remains: pulsar beams show subpulse phase reversals at the longitude-stationary boundaries separating individual pulse components. We have shown that these changes are comprehensible in the context of an 
oblique pulsator model incorporating non-radial pulsations of high degree $\ell$. The important features of our model are: non-radial oscillations aligned to and symmetric about the pulsar magnetic axis; surface displacements that follow a spherical harmonic distribution; radio emission that follows the displacements but is never negative; pulsation modes of sufficiently high $\ell$ that nodal lines often appear in the pulse window; and pulsation frequencies that remain coherent over many pulsar spin periods. Variations on this basic model might include multiple pulsation modes with non-zero azimuthal orders, pulsations that are distorted, in reality or in appearance, by non-dipole fields, and modes that interact either through mode coupling or a non-linear emission mechanism.

Our model qualitatively reproduces the mean shapes of pulsar beams and the radio frequency dependent behavior of subpulses with a minimum of free parameters. In the most basic form of the model these are $\alpha, \beta, P_{\text {time }}, \ell$, and $P_{1}$. Our model also dictates specific requirements that can be tested quantitatively using new or archival data. We have embarked on a program to conduct such tests and we encourage others to do likewise. If the model survives these tests, then we will have the opportunity to measure fundamental properties of matter in a domain not accessible to laboratory experiments. The first challenge will be to determine the site of the pulsations, and then to connect measured eigenfrequencies with the eigenmodes of a structural model. Given the number of modes in the pulsation spectrum at large $\ell$, this may be a daunting task, but even rough identification will provide limits on the thermal, electrical, and mechanical properties of constituents of a neutron star, the densest objects accessible to direct observational scrutiny.

We are grateful to Chuck Evans and Don Winget for helpful conversations, and to Joanna Rankin for providing the archival data on PSR0943+10. One of us is grateful to Gus and Jack for providing a quiet environment for the completion of this work. This work was supported by a CAREER grant from the National Science Foundation (AST 000-94289) and by a fellowship from the Alfred P. Sloan Foundation.

\section{A. MODULATION INDUCED DRIFTBAND CURVATURE}

The purpose of this appendix is to calculate, in approximate fashion, the apparent change in subpulse drift between nodal lines caused by the amplitude modulation of strictly periodic time-like pulses. We will approximate the longitude-dependent modulation between two nodes as a cosine function, which is very similar to the envelope between two nodes of a spherical harmonic for sightlines with $\beta=0$. Thus we can write a more manageable version 
of equation 1:

$$
\xi=\cos \left(\omega_{\Phi} t\right) \cos \left(\omega_{t} t+\phi\right),
$$

where $\omega_{\Phi}=\frac{2 \pi}{P_{\text {node }}}$.

To find the times of maxima (and minima), we take the derivative and set it to zero, yielding:

$$
\frac{-\omega_{\Phi}}{\omega_{t}+\phi}=\frac{\tan \left(\omega_{t} t+\phi\right)}{\tan \left(\omega_{\Phi} t\right)} .
$$

In figure 9 we have plotted the locus of the times-of-maxima for $\omega_{\Phi} t$ between -90 and 90 degrees, simulating the range between nodal lines, for a variety of cases. The lines in these plots are analogous to subpulse driftbands, because they show how the maxima (and minima) of time-like pulses vary with longitude. For the case where $P_{\text {time }} \approx P_{\text {node }}$, we expect no driftband curvature. When $P_{\text {time }}<P_{\text {node }}$, we expect slower drift near nodal lines (right hand panels), and vice versa.

We note that for $\beta \neq 0$ sightlines that graze along a nodal line in the center of the pulse window, the driftband curvature can be the reverse of the cases plotted here, i.e. narrow subpulses will drift more slowly near the center of the profile. This can explain the driftband curvature measured for PSR0031-07 by Krishnamohan (1980), and discussed by Wright (1981). The more important conclusion is that when nodal lines are present, $P_{2}$ can vary with longitude, even though the underlying clock is absolutely stable.

\section{REFERENCES}

Backer, D. C. 1970a, Nature, 228, 42

Backer, D. C. 1970b, Nature, 228, 1297

Backer, D. C. 1970c, Nature, 227, 692

Backer, D. C. 1976, ApJ, 209, 895

Bartel, N., Morris, D., Sieber, W., \& Hankins, T. H. 1982, ApJ, 258, 776

Biggs, J. D. 1990, MNRAS, 246, 341

Fig. 9.- Simulated driftband curvature calculated from equation A2 for cases where $P_{\text {time }}=$ $P_{\text {node }}$ (top), $P_{\text {time }}>P_{\text {node }}$ (left) and $P_{\text {time }}<P_{\text {node }}$ (right). 
Boriakoff, V. 1976, ApJ, 208,L43

Carroll, B. W., Zweibel, E. G., Hansen, C. J., McDermott, P. N., Savedoff, M. P., Thomas, J. H., \& Van Horn, H. M. 1986, ApJ, 305, 767

Clemens, S. L. 1883, Life on the Mississippi, 17

Deshpande, A. A., \& Rankin, J. M. 2001, ApJ, 322, 438

Drake, F. D., \& Craft, H. D. 1968, Nature, 220, 231

Duncan, R. C. 1998, ApJ, 498, L45

Dziembowski, W. 1982, Acta Astronomica, 32, 147

Edwards, R. T., \& Stappers, B. W. 2002, A\&A, 393, 733

Edwards, R. T., \& Stappers, B. W. 2003, A\&A, 410, 961

Edwards, R. T., Stappers, B. W., \& van Leeuwen, A. G. J. 2003, A\&A, 402, 321

Finn, L. S. 1990, MNRAS, 245, 82

Fowler, L. A., Wright, G. A. E., \& Morris, D. 1981, A\&A, 93, 54

Gangadhara, R. T., \& Gupta, Y. 2001, ApJ, 555, 31

Gil, J. A. 1991 A\&A, 243, 219

Gil, J., \& Kijak, J. 1992, A\&A, 256, 477

Gil, J. A., Kijak, J., \& Seiradakis, J. H. 1993, A\&A, 272, 268

Gil, J., Gupta, Y., Gothoskar, P. B., \& Kijak, J. 2002, ApJ, 565, 500

Gold, T. 1969, Nature, 221, 25

Goldreich, P., \& Julian, W. H. 1969, ApJ, 157, 869

Gould, D. M 1994, PhD Thesis, Jodrell Bank Observatory

Graham-Smith, F. 2003, Rep. Prog. Phys., 66, 173

Gupta, Y., \& Gangadhara, R. T. 2003, ApJ, 584, 418

Han, J. L., \& Manchester, R. N. 2001, MNRAS, 320, L35 
Hankins, T. H., \& Wolszczan, A. 1987, ApJ, 318, 410

Hankins, T. H., \& Wright, G. A. E. 1980, Nature, 288, 681

Hansen, C. J., \& Cioffi, D. F. 1980, ApJ, 238, 740

Hewish, A. 1970, ARAA, 8, 265

Hewish, A., Bell, S. J., Pilkington, J. D. H., Scott, P. F., \& Collins, R. A. 1968, Nature, 217, 709

Izvekova, V. A., Kuzmin, A. D., Lyne, A. G., Shitov, Y. P., \& Graham- Smith, F. 1993, MNRAS, 261, 865

Jessner, A., Lesch, H., \& Kunzl, T. 2001, ApJ, 547, 959

Kleinman, S. J., et al. 1998, ApJ, 495, 424

Komesaroff, M. M. 1970, Nature, 225, 612

Komesaroff, M. M., Morris, D., \& Cooke, D. J. 1970, Astrophys. Lett., 5, 37

Kramer, M., Wielebinski, R., Jessner, A., Gil, J. A., \& Seiradakis, J. H. 1994, A\&AS, 107, 515

Krishnamohan, S. 1980, MNRAS, 191, 237

Kurtz, D. W. 1982, MNRAS, 200, 807

Kurtz, D. W., Shiahashi, H., \& Goode, P. R. 1990, MNRAS, 247, 558

Lesch, H., Jessner, A., Kramer, M., \& Kunzl, T. 1998, A\&A, 332, L21

Lyne, A. G., \& Ashworth, M. 1983, MNRAS, 204, 519

Lyne, A. G., \& Manchester, R. N. 1988, MNRAS, 234, 477

Lyne, A. G., Smith, F. G., \& Graham, D. A. 1971, MNRAS, 153, 337

McDermott, P. N., van Horn, H. M., \& Hansen, C. J. 1988, ApJ, 325, 725

Manchester, R. N. 1995, JA\&A, 16, 107

Melrose, D. B. 1995, JA\&A, 16, 137

Mitra, D., \& Deshpande, A. A. 1999, A\&A, 346, 906 
Mitra, D., \& Rankin, J. M. 2002, ApJ, 577, 322

Oster, L., \& Sieber, W. 1977, A\&A, 58, 303

Potekhin, A. Y. 1999, A\&A, 351, 787

Prószyński, M., \& Wolszczan, A. 1986, ApJ, 307, 540

Radhakrishnan, V., \& Cooke, D. J. 1969, Astrophys. Lett., 3, 225

Radhakrishnan, V., \& Rankin, J. M. 1990, ApJ, 352, 258

Rankin, J. M. 1983a, ApJ, 274, 333

Rankin, J. M. 1983b, ApJ, 274, 359

Rankin, J. M. 1986, ApJ, 301, 901

Rankin, J. M. 1990, ApJ, 352, 247

Rankin, J. M. 1993a, ApJ, 405, 285

Rankin, J. M. 1993b, ApJS, 85, 145

Reisenegger, A., \& Goldreich, P. 1992, ApJ, 395, 240

Richardson, M. B., van Horn, H. M., Ratcliff, K. F., \& Malone, R. C. 1982, ApJ, 255, 624

Rickett, B. J. 1975, ApJ, 197, 185

Robinson, E. L., Kepler, S. O., \& Nather, R. E. 1982, ApJ, 259, 219

Ruderman, M. A. 1968, Nature, 218, 1128

Ruderman, M., \& Sutherland, P. G. 1975, ApJ, 196, 51

Rylov, I. A. 1978, Ap\&SS, 53, 377

Schopper, R., Ruhl, H., Kunzl, T. A., \& Lesch, H. 2002, Neutron Stars, Pulsars, and Supernova Remnants, 193

Smith, F. G. 1970, MNRAS, 149, 1

Staelin, D. H., Ewing, M. S., Price, R. M., \& Sutton, J. M. 1970, ApJ, 160, L7

Strohmayer, T. E. 1992, ApJ, 388, 138 
Strohmayer, T. E., Cordes, J. M., \& van Horn, H. M. 1992, ApJ, 389, 685

Strohmayer, T. E. 1993, ApJ, 417, 273

Suleymanova, S. A., Izvekova, V. A., Rankin, J. M. \& Rathnasree, N. 1998, JA\&A, 19, 1

Taylor, J. H., Manchester, R. N., \& Huguenin, G. R. 1975, ApJ, 195, 513

Thorsett, S. E. 1991, ApJ, 377, 263

Wright, G. A. E. 1981, MNRAS, 196, 153

Wu, Y., \& Goldreich, P. 2001, ApJ, 546, 469

Van Horn, H. M. 1980, ApJ, 236, 899

Van Leeuwen, A. G. J., Kouwenhoven, M. L. A., Ramachandran, R., Rankin, J. M., \& Stappers, B. W. 2002, A\&A, 387, 169

Van Leeuwen, A. G. J, Stappers, B. W., Ramachandran, R., \& Rankin, J. M. 2003, A\&A, 399,223

Winget, D. E., van Horn, H. M., \& Hansen, C. J. 1981, ApJ, 245, L33

Zheleznyakov, V. V. 1971, ApSS, 13, 87 
This figure "f1.png" is available in "png" format from: http://arxiv.org/ps/astro-ph/0403317v1 
This figure "f2.png" is available in "png" format from: http://arxiv.org/ps/astro-ph/0403317v1 
This figure "f3.png" is available in "png" format from: http://arxiv.org/ps/astro-ph/0403317v1 
This figure "f4.png" is available in "png" format from: http://arxiv.org/ps/astro-ph/0403317v1 
This figure "f5.png" is available in "png" format from: http://arxiv.org/ps/astro-ph/0403317v1 
This figure "f6.png" is available in "png" format from: http://arxiv.org/ps/astro-ph/0403317v1 
This figure "f7.png" is available in "png" format from: http://arxiv.org/ps/astro-ph/0403317v1 
This figure "f8.png" is available in "png" format from: http://arxiv.org/ps/astro-ph/0403317v1 
This figure "f9.png" is available in "png" format from: http://arxiv.org/ps/astro-ph/0403317v1 